\title{
In-Situ Characterization of Deposits in Ceramic Hollow Fiber Membranes by Compressed Sensing RARE-MRI
}

\author{
S. Schuhmann, N. Schork, K. Beller, and H. Nirschl \\ Karlsruhe Institute of Technology (KIT), Institute of Mechanical Process Engineering and Mechanics, Karlsruhe \\ 76131, Germany \\ T. Oerther \\ Bruker Biospin GmbH, Rheinstetten 76287, Germany \\ G. Guthausen \\ Karlsruhe Institute of Technology (KIT), Institute of Mechanical Process Engineering and Mechanics, Karlsruhe \\ 76131, Germany \\ Karlsruhe Institute of Technology (KIT), Chair of Water Chemistry and Water Technology, Karlsruhe 76131, \\ Germany
}

\begin{abstract}
Ultrafiltration with ceramic hollow fiber membranes was investigated by compressed sensing rapid acquisition relaxation enhancement (CS-RARE) magnetic resonance imaging (MRI) to characterize filtration mechanisms. Sodium alginate was used as a model substance for extracellular polymeric substances. Dependent on the concentration of divalent ions like $\mathrm{Ca}^{2+}$ in an aqueous alginate solution, the characteristics of the filtration change from concentration polarization to a gel layer. The fouling inside the membrane lumen could be measured by MRI with a CS-RARE pulse sequence. Contrast agents have been used to get an appropriate contrast between deposit and feed. The lumen was analyzed quantitatively by exploring the membrane's radial symmetry, and the resulting intensity could be modeled. Thus, different fouling mechanisms could be distinguished. CS-RARE-MRI was proven to be an appropriate in situ tool to quantitatively characterize the deposit formation during in-out filtration processes. The results were underlined by flux interruption experiments and length dependent studies, which make it possible to differentiate between gel layer or cake filtration and concentration polarization filtration processes.
\end{abstract}

Keywords: fouling, alginate, NMR imaging, ceramic hollow fiber membranes, compressed sensing

\section{Introduction}

Ceramic hollow fiber membranes (CHFM) are used for ultra filtration because of their resistance against high temperatures and chemicals. They have a wide range of applications and are used for example in food, pharmaceutical, and water industries. The CHFMs in this publication are operated in in out filtration mode, which leads to higher shear rates on the inside of the CHFM compared to out in filtrations. ${ }^{1}$ CHFMs can be used in two operation modes: dead end and cross flow filtration. The operation mode depends on the nature of feed and application. Dead end filtration is applied at low feed concentrations whereas cross flow filtrations are usually used for higher con centrations to realize higher shear forces on the deposits due to the parallel flow. ${ }^{2}$ This article focuses on dead end filtrations.

Sodium alginate is often used as a model substance for extracellular polymeric substances, which makes experiments

Correspondence concerning this article should be addressed to S. Schuhmann at sebastian.schuhmann@kit.edu. comparable and reproducible. The filtration mechanism depends on the feed composition. Literature distinguishes between cake filtration, concentration polarization, and gel layer filtration. ${ }^{3}$ In the filtration of aqueous solutions of sodium alginate, concentration polarization was observed. The properties of the sodium alginate feed can be changed by add ing divalent ions to the feed, for example, in form of $\mathrm{CaCl}_{2}$. Sodium alginate will then form a gel. This characteristic of sodium alginate allows to in situ study these two different fil tration mechanisms by MRI noninvasively.

MRI is known in medical applications, but also explored in technical and scientific processes and filtration. ${ }^{4-17}$ In this arti cle, MRI is used for the in situ, time resolved observation of the filtration progress in CHFM lumen. Especially the time evolution and the nature of the deposit is of major interest. The MRI measurement time depends on the pulse sequence, the resolution and the desired signal to noise ratio of the images. Due to the time evolution of the filtration process, comparatively short measurement times are essential. Com pressed sensing (CS) allows to reduce the measurement time drastically. Nearly the same image quality can be achieved by 
CS reconstruction, ${ }^{18}$ which permits the observation of the fil tration's progress with an adequate time resolution.

The approach in the present article is to elucidate the filtra tion mechanisms in CHFMs dependent on the composition of the feed solution by CS RARE MRI. Two extremes were stud ied to develop a quantitative description of deposit formation for concentration polarization and gel layer formation with an adequate spatial and time resolution.

\section{Experimental}

\section{Feed solutions with contrast agents}

An aqueous sodium alginate solution (Alginic acid sodium salt from brown algae "medium viscosity" from Sigma Aldrich) was used as a model substance for extracellular poly meric substances. The concentration of sodium alginate was $200 \mathrm{mg} / \mathrm{L}$. Sodium alginate is known to form gel structures in the presence of divalent ions like $\mathrm{Ca}^{2+}$. In former experi ments, loose polarization layers or gel layers could be observed. ${ }^{19-21} \mathrm{Ca}^{2+}$ ions were added in form of $\mathrm{CaCl}_{2}$ (Sigma Aldrich) with a concentration of $c_{\mathrm{Ca}^{2+}} 2 \mathrm{mmol} / \mathrm{L}$. Due to the poor contrast between alginate and water a contrast agent (Magnetite Alginate 100 MagAlg100, also named MION $)^{22}$ was added to the feed solution in concentrations of $1 \mathrm{mM}$ and below. These MIONs show predominately a $T_{2}$ con trast, the regions with significant concentration of the MIONs will therefore appear with lower intensities in relaxation weighted MRI. Long term MRI experiments proved that MIONs in the used concentration have no influence on the fil tration mechanism. Also the permeate flux did not change by adding MIONs to the feed solution. Additional experiments revealed the following behavior of MIONs which is essential in the interpretation of the MRI data: When the MIONs are dissolved in water, they tend to sediment. When MIONs were dissolved in an alginate solution, they diffuse together with the alginate without sedimentation. Dissolved in an alginate gel, the MIONs don't migrate out of the gel into the surround ing water. Thus, these MIONs can be used to increase the MRI contrast by paramagnetic relaxation enhancement.

\section{Hollow fiber membrane}

For the ultrafiltration in wastewater treatment, in out filtra tion hollow fiber membranes were used as provided by MAN $\mathrm{N}+$ HUMMEL GmbH, Ludwigsburg, Germany. The fibers with an inner lumen diameter of about $1.9 \mathrm{~mm}$ and an outer fiber diameter of $3.2 \mathrm{~mm}^{20,23}$ are made of porous $\mathrm{Al}_{2} \mathrm{O}_{3}$ ceramics. The hollow fiber membranes have a radially asym metric structure showing different layers along the radius. The outer and highly porous layer is responsible for the mechanical stability and the form of the hollow fiber whereas the inner layer is responsible for the filtration characteristics. This inner active layer has an average pore diameter of $40 \mathrm{~nm}$ and thick ness in the order of $20 \mu \mathrm{m}$. CHFMs have the ability to with stand high temperatures and are tolerant against aggressive chemical cleaning. A water permeability between $170 \mathrm{~L} /\left(\mathrm{m}^{2} \mathrm{~h}\right.$ bar) and $315 \mathrm{~L} /\left(\mathrm{m}^{2} \mathrm{~h}\right.$ bar) could be measured on the used ceramic hollow fiber membranes.

\section{In situ filtration experiments}

Filtration experiments were performed in a single fiber fil tration setup (Figure 1). The filtration module was investigated using a Bruker Avance HDIII SWB $200 \mathrm{MHz}$ spectrometer equipped for MRI. ${ }^{19-21}$ The probe was a $20 \mathrm{~mm}$ birdcage of

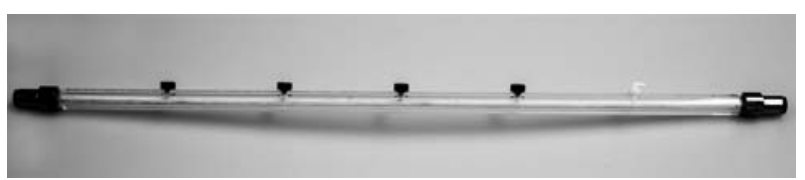

Figure 1. Single fiber filtration module which is mounted in the tomograph. Multiple permeate outlets are available, but only one is used in the experiments.

the MICWB40 series. This allows for an in situ observation of the membrane lumen during the filtration. Due to the module length of $420 \mathrm{~mm}$, the module was observed in a first experi ment only in a segment of $25 \mathrm{~mm}$ near the permeate outlet. Shifting the module during the filtration in a second experi ment allows a limited but meaningful observation of the deposits along its length axis ( $z$ in the following) of the hollow fiber membrane. The feed solution was pumped into the mod ule by a pressure vessel applying a transmembrane pressure of 1 bar leading to typical permeate volume flow rates of 1 $5 \mathrm{~mL} / \mathrm{min}$ which corresponds to laminar flow. By closing the valve of the retentate outlet, a dead end filtration can be per formed. The permeate volume was measured volumetrically over time.

\section{MRI methods}

A fast spin echo method is needed for in situ filtration in CHFMs because of the inherent $T_{2}$ contrast which is generated by the contrast agent but also because of large susceptibility differences between liquids and ceramics. ${ }^{19}$ A RARE pulse sequence was used to measure the deposit formation in the membrane lumen while exploring the possibilities of CS. A series of $180^{\circ}$ pulses after a $90^{\circ}$ excitation pulse generates a series of echoes with different phase encodings but also with different transverse relaxation weighting. Multiples lines of $k$ space are measured during a given repetition time $T_{R}$. The number of echoes which is collected by one $T_{R}$ is named RARE factor $R F .^{24} R F$ has a significant influence on the images, conversely RARE is known to be sensitive to move ments, that is, in our case to velocities in the feed lumen and in the permeate. Specifically, the inflow effect should be men tioned. Low $R F$ and large $T_{R}$ diminish this effect, which makes it hard to reduce the measurement time with commonly used NMR pulse sequence parameters. However, the time resolu tion of the MRI experiments has to be much smaller than the filtration progress. For example, at the beginning of the filtra tion, the feed velocities change very fast on the subminute time scale as the volume flow rate does. Short measurement times are therefore mandatory to detect the formation of the deposit layer spatially and time resolved. To reduce the mea surement time without reinforcing the inflow effect or reduc ing the spatial resolution or signal to noise ratio, a CS RARE sampling pattern was used with $50 \%$ of the full phase encod ing steps (Figures 2a, b).

A Box Müller method was applied to obtain the sampling scheme with a standard deviation $\sigma \quad 0.33 .^{25}$ Fifty percent of the points were mainly sampled in the $k$ space center along the phase encoding direction. The read direction ( $x$ direction) was sampled completely. The image reconstruction was performed with a $l_{1}$ penalized nonlinear conjugate gradient reconstruction solving the following optimization problem 
a)
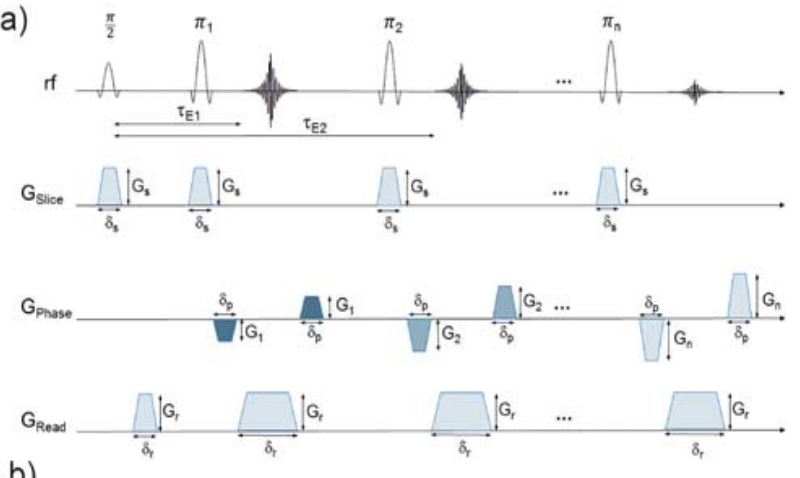

b)

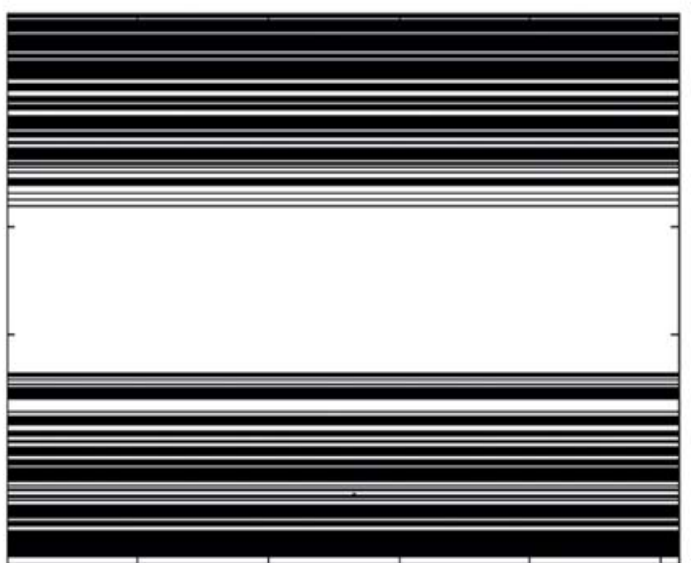

Figure 2. (a) RARE sequence and (b) CS-RARE sampling scheme: Only $50 \%$ of the phase encoding steps were measured ( $y$-direction) to reduce the measurement time. All 256 points were measured in read direction ( $x$-direction). The phase encoding steps were obtained by applying a Box-Müller sampling pattern.

[Color figure can be viewed at wileyonlinelibrary.com]

$$
\operatorname{argmin}_{S}\left\{\|F S \quad K\|_{2}+\lambda_{1}\|S\|_{1}+\lambda_{2} T V(S)\right\}
$$

$F$ is the Fourier operator applied to the image $S, K$ describes the measured data in $k$ space, $\lambda_{1,2}$ are the regularization parameters, and $T V$ is the Total Variation. The reconstruction was performed with a modified MATLAB script available in Ref. 26. Due to the CS sampling pattern the measurement time was reduced to $3 \mathrm{~min} 12 \mathrm{~s}$ with pulse sequence parameters summarized in Table 1.

\section{Quantification of the deposit layers}

To characterize the fouling layer, the cylindrical geometry of the hollow fiber membrane was explored. Concentric rings

Table 1. MRI Parameters of the CS-RARE Pulse Sequence

\begin{tabular}{lc}
\hline MRI Parameter & Value \\
\hline$T_{R}$ & $3 \mathrm{~s}$ \\
$\tau_{E, \text { ieffective }}$ & $5.8 \mathrm{~ms}$ \\
Field of view & $8 \times 8 \mathrm{~mm}$ \\
slice thickness & $2 \mathrm{~mm}$ \\
no. pixels & $256 \times 256$ \\
in plane resolution & $31.25 \mu \mathrm{m}$ \\
RARE factor & 2 \\
CS undersampling & $50 \%$ \\
measurement time & $3 \mathrm{~min} 12 \mathrm{~s}$ \\
\hline
\end{tabular}

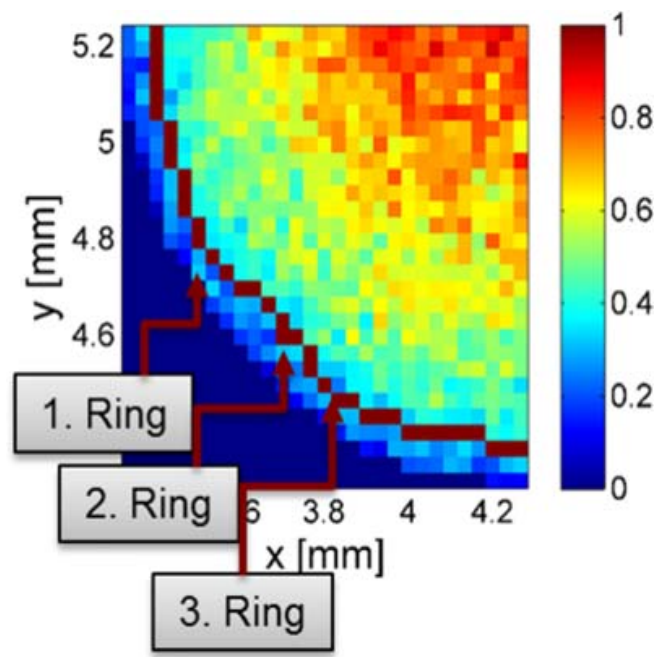

Figure 3. Scheme for determination of the radial intensity distribution: starting from the membrane wall an arbitrarily shaped ring is identified by the intensity jump between wall and feed in the beginning of filtration. The consecutive rings are placed concentrically and selfsimilar with decreasing radius toward the center of the lumen.

[Color figure can be viewed at wileyonlinelibrary.com]

were inscribed into the lumen from the membrane wall toward the center of the lumen (Figure 3).

The pixel intensities in this ring, which adapts the inner shape of the membrane, are averaged, and the mean intensity is obtained as a function of the radius in the lumen. Conven tionally the radius is defined starting in the center of the lumen. Thus, despite of the procedure starting at the mem brane's inner wall, the radial MRI intensity distribution is cal culated with $I(0)$ being the intensity in the center of the lumen. By visual inspection of the MRI images it was confirmed that the deposit was accumulating uniformly and symmetrically during the filtration processes, that is, no angular dependence of the deposit was observed. The average over all angles was thus found to be a good assumption. In a next step the radial intensity distribution was quantified as a function of time. The intensity gradient from the membrane center to the mem brane's inner surface can thus be detected and modelled in terms of filtration theory. During the filtration, the module can additionally be shifted to different $z$ positions to measure the axial dependence of deposition.

\section{Modelling of the MRI intensity distribution}

Concentration profiles in the membrane lumen were described in literature. ${ }^{3,27}$ A model was derived under station ary conditions by considering the convective and diffusive mass transport. Under the additional assumption that the dis solved molecules, here alginate and the contrast agent, do not pass the membrane, their concentration $c_{S}$ as a function of a spatial coordinate can be modelled by an exponentially increasing function

$$
\frac{C_{S}}{C_{s, \text { feed }}} \exp \left(\frac{J_{V} r}{D}\right)
$$

The quotient of the spatially varying concentration $c_{S}$ and the feed concentration $c_{s \text {, feed }}$ is an exponential function of the flux $J_{V} \quad \frac{d V}{A d t}$, that is, the differential permeate volume $V$ per time 
interval and filtration area $A$, the diffusion coefficient $D$, and the radial coordinate $r$. The equation results from consider ations of mass balance and Fick's law of diffusion.

In the case of in situ MRI experiments, the alginate macro molecules together with the contrast agent accumulate at the membrane surface, which leads to lower MRI intensities, that is, negative image contrast, due to the dominant relaxivity $r_{2}$ of the MIONs. As a function of filtration time, the region increases where lower intensities are observed. Assuming that the model in Eq. 2 is reasonable also in the case of the non equilibrium conditions in MRI, that is, nonvanishing permeate flux, an empirical approach is chosen to describe the radial growth of the deposit: An exponential increase of concentra tion toward the membrane surface is assumed. Reformulating Eq. 2 in terms of MRI relevant quantities in an ongoing filtra tion, the radially averaged MRI intensity $I(r)$ can be written as

$$
\frac{I(r, t)}{I(0)} \quad 1 \quad B \exp \left(\frac{r}{h(t)}\right)
$$

$h(t)$ is the time dependent size of the deposit and $I(0)$ the NMR intensity of the feed solution, $B$ is a numerical factor for improving the fit quality. The value of $B$ is between 0 and 1 and describes the amount of deposit. Care was taken to avoid inflow artefacts to guarantee an appropriate reference intensity $I(0)$ in the lumen. The enrichment of alginate, that is, MIONs, toward the membrane wall leads to an intensity reduction, also leading to the modification of the mathematical expression of Eq. 3 when compared with Eq. 2. The center of the coordinate system is located in the center of the hollow fiber with $r \quad 0$. Thus, the radial symmetry of the CHFM can be exploited by averaging over the angle in the axial images while retaining the radial spatial resolution to derive the deposit height $h(t)$. Please note that $h$ (Eq. 3) is defined in this context as the length at which the intensity decreased by a factor of $1 / e$ with respect to the feed value. $h$ does not represent the total height of the deposit although being related to this quantity. The geo metric characteristics of the fouling layer is given by $h$ within this description.

With progressing filtration time, the deposit will grow. The analysis of $h(t)$ as a function of filtration time $t$ results in detailed information on an in out ultrafiltration process regard ing deposit formation. The simplest approach is an exponen tial, which is modeled in the present case by the commonly known exponential growth function (4) describing the data well within the experimental error

$$
h(t) \quad h_{0}\left(1 \exp \left(\frac{t}{t_{c}}\right)\right)
$$

As a result, the time constant $t_{c}$, describes the build up of the deposit. $t_{c}$, depends on the filtration process.

\section{Model for gel relaxation and equilibration of concentration gradients}

A common definition to distinguish gel, that is, fouling layers, from concentration polarization is the characteristics when releasing the filtration pressure and subsequently starting a second filtration. While a gel can relax and swell, a concen tration gradient as proposed in the case of pure concentration polarization will equilibrate according to Fick's law when stopping the filtration process. Macroscopically, the different filtration processes can be differentiated accordingly by recording the flux after relaxation and equilibration of the deposit in a second filtration. In the case of concentration polarization, $J_{V}$ will return to approximately the value of the native fiber, whereas a continuation with the small values at the end of the first filtration will be observed in the case of cake or dense gel layers. ${ }^{28}$

The gel compressibility and swelling can be observed by MRI. It was empirically modeled similar to Eq. 3 but applying a Gaussian function revealing the Gaussian width $h_{\mathrm{G}}$ as a func tion of swelling, that is, equilibration. Again, the time depen dence of $h_{\mathrm{G}}$ can be described by an exponentially growing function according to Eq. 4. This approach reveals the charac teristic gel relaxation time on a sudden pressure release.

In the case of pure concentration polarization, the time scale is rather short given by Fick's law of diffusive concentration equilibration. It was found that the time needed for pressure release and the subsequent MRI experiment is already too large in the present case to allow for a quantitative modeling of the concentration equilibration. Already after one image acquisition ( $3 \mathrm{~min} 12 \mathrm{~s}$ ) after pressure release, the concentra tion polarization has almost completely disappeared. The question to be answered in this case therefore concerns the degree of reversibility which was addressed by repeating the filtration.

\section{Results and Discussion \\ Quantification of deposition and impact of the filtration mechanisms as measured by MRI}

CS RARE MRI images allow to detect the membrane lumen in situ during the in out filtration. With progressing filtration time, an increasing deposit layer is observed on the mem brane's inner surface. It appears with low intensities caused by the dominantly negative $T_{2}$ contrast induced by MagAlg100 as was shown earlier. ${ }^{19,20}$ The CS sampling pattern allows faster measurements with same spatial resolution but lower inflow artifacts as the repetition time $T_{R}$ can be chosen ade quately high to avoid inflow effects without losing the required time resolution for observing the progress of the filtration pro cess. The quantification of the deposit height and the time scale was performed from the time series of MRI experiments as described above.

Filtration of Sodium Alginate with $\mathrm{Ca}^{2+}$ : Gel Formation and Compressibility. A filtration using a transmembrane pressure of 1 bar was performed with the described sodium alginate solution while axial CS RARE MRI images were acquired (Figure 4a). A small inflow artefact is visible in the permeate channel, which however does not contribute to the quantification in the feed channel. This is due to the vanishing MION concentration in the permeate leading to longer longitu dinal relaxation when compared with the feed solution. The inflow effect could be avoided by longer repetition times, which, however, would lead to inadequately time resolution. The normalized intensities were calculated as a function of the radial distance from the membrane wall (Figure $4 b$ ). Equation 3 was fitted to the data and reveal a typical time evolution of the deposit layer characterized by the parameter $h(t)$. The growth of the deposit layer in terms of $h(t)$ was modeled by Eq. 4 (Figure $4 \mathrm{c}$ ) revealing the growth time constant $t_{c}$ or its reciprocal value, the growth rate $r_{c} \quad 1 /(482 \mathrm{~min}) \quad 0.125 / \mathrm{h}$.

After interrupting the filtration, the fouling layer remains sticking to the membrane surface. The fouling layer even increases its size, which is a sign of a swelling gel: When the pressure is released the compressible gel will swell, which leads to a dilation of the fouling layer. This underlines the 
a)

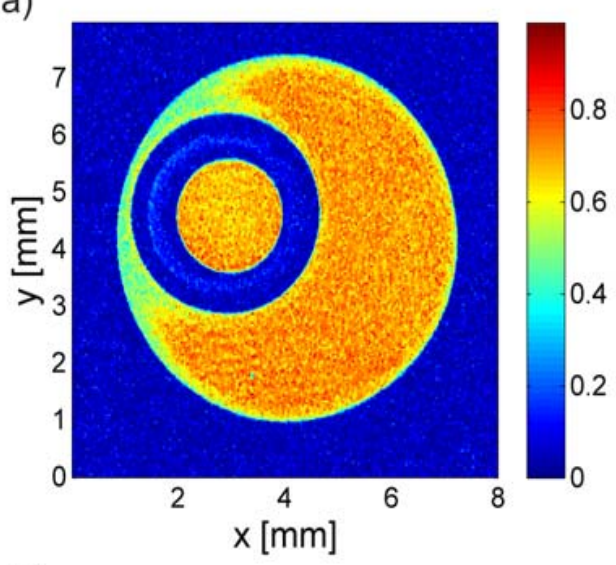

b)

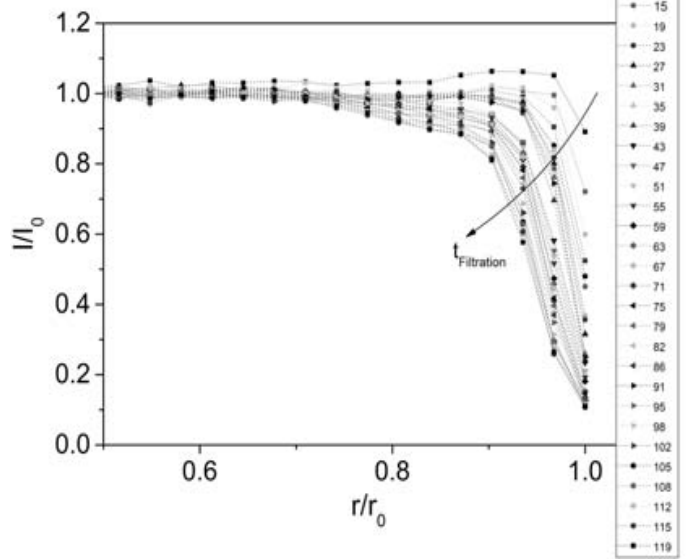

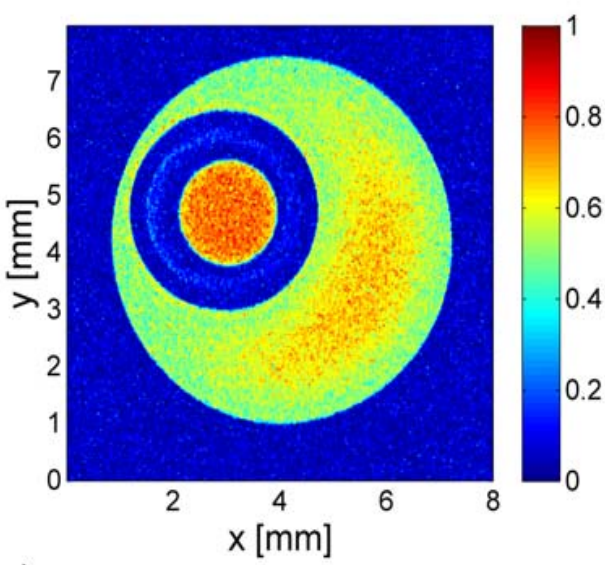

c)

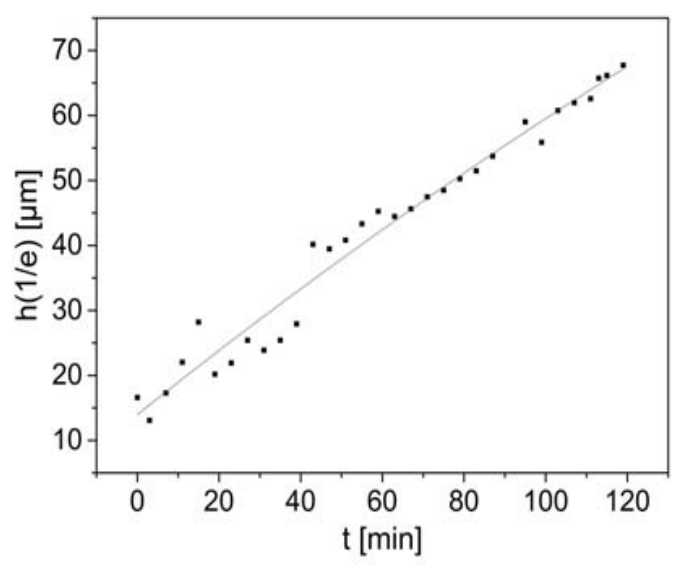

Figure 4. (a) Axial images at start and end of an ultrafiltration of aqueous sodium alginate solution with $\mathrm{Ca}^{2+}$. (b) Radial intensity profiles along the filtration process from which the height $h$ of the deposit layer was calculated. (c) Time evolution of $\boldsymbol{h}$ and modeling by an exponential growth function.

[Color figure can be viewed at wileyonlinelibrary.com]

statement of a gel layer model. The time constant $t_{c}$ for swell ing of the gel layer amounted to 16 min according to Eq. 4. After the interruption, a second filtration was performed with same filtration parameters under in situ observation by MRI. In this second filtration period, the fouling layer continues to build up on the 'old' fouling layer of the first filtration interval which still sticks on the membrane surface. The flux is found to continuously decrease further without any step as observed in earlier experiments.

Filtration of Sodium Alginate Without $\mathrm{Ca}^{2+}$ : Concentra tion Polarization and Equilibration. A loose layer can be observed in the filtration of sodium alginate without $\mathrm{Ca}^{2+}$ as described previously. ${ }^{19,20}$ The MRI data were quantitatively analyzed to characterize the filtration process in more detail by applying the mentioned angular average (Figure 5a). The data points slightly oscillate due to MRI data acquisition arte facts. However, the fit of Eq. 3 to the data is numerically suffi ciently stable to compensate for this imperfection. As a result, $h(t)$ obviously depends on the filtration time (Figure 5b) and is much larger than in the case of alginate filtration without $\mathrm{Ca}^{2+}$. When fitting Eq. 4 to the data, the time scale $t_{c}$ is deter mined, describing the deposition process during filtration. For the filtration without $\mathrm{Ca}^{2+}, t_{c}$ amounts to $14.516 \mathrm{~min}$ com pared to $t_{c} 484$ min for the filtration with $\mathrm{Ca}^{2+}$. These find ings confirm that the filtration mechanisms differ and depend on the concentration of the divalent ions. $h(t)$ quantitatively describes the concentration polarization, that is, a concentra tion gradient from the feed channel in the middle of the lumen toward the membrane's inner surface in the present case of fil tration without $\mathrm{Ca}^{2+}$. The smaller values for $t_{c}$ are a hint for a tendency to build up a polarization concentration. Higher val ues of $t_{c}$ will indicate a more gel layer like filtration.

After $90 \mathrm{~min}$, the filtration was stopped, and the pressure was set to zero to observe the relaxation of the deposit in anal ogy to the experimental design for the filtration with $\mathrm{Ca}^{2+}$. Due to pressure release, the signal intensities in the membrane lumen for the filtration without $\mathrm{Ca}^{2+}$ equilibrate. This is another indication for concentration polarization. A different behavior was observed compared to the filtration with $\mathrm{Ca}^{2+}$.

An important question concerns the degree of reversibility of filtration. Apart from the technical and practical issues related to this question, the answer also influences the model ing of the filtration process itself. In the case of a pure concen tration polarization the reversibility should be measurable either by repeated filtration cycles in MRI or by time resolved measurements of the classic integral values of flux to be dis cussed in the next section. After the first filtration and the equilibration of the MRI intensities in the feed channel, a sec ond and third filtration were performed subsequently with MRI measurements at the same position of the fiber. Between 

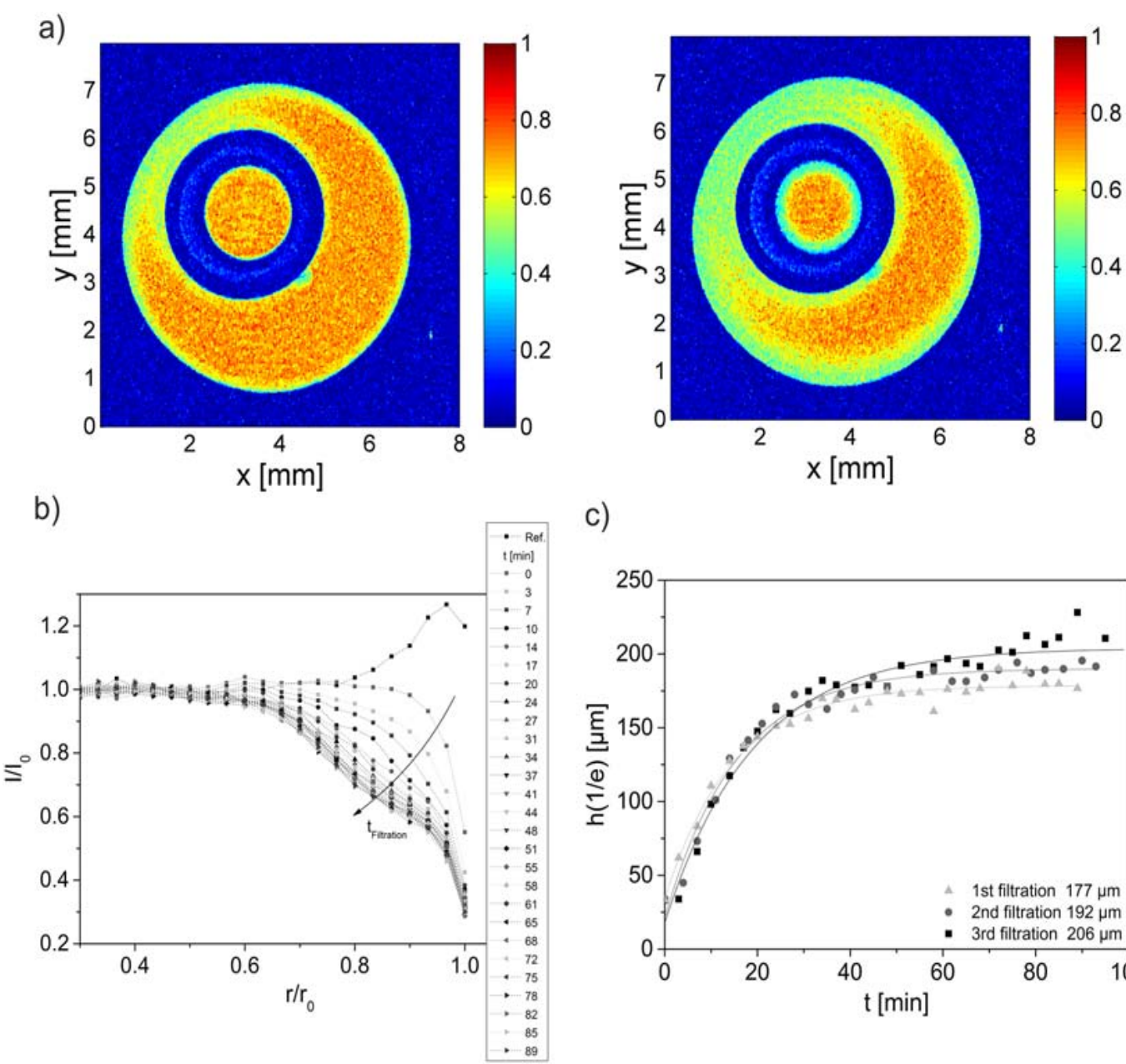

C)

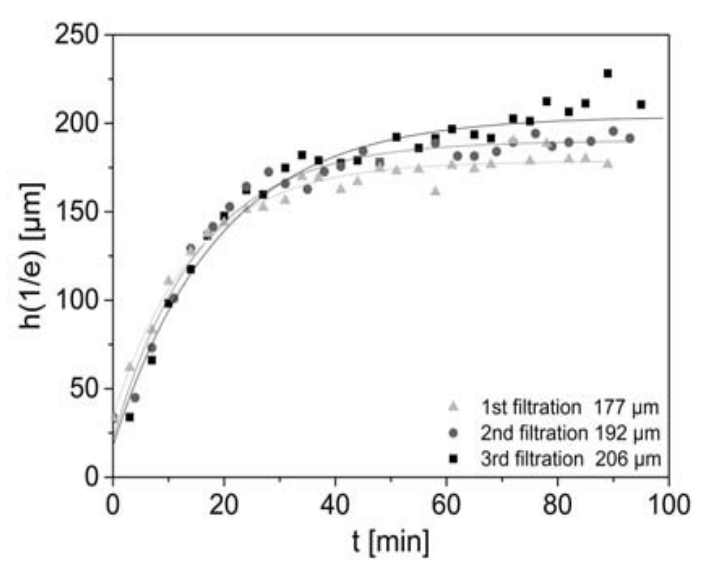

Figure 5. (a) Axial images at start and end of an ultrafiltration of aqueous sodium alginate solution without $\mathrm{Ca}^{2+}$. (b) Radial intensity profiles along the filtration process from which the height $\boldsymbol{h}$ of the deposit layer was calculated. (c) Time evolution of $h$ and modeling by a much faster exponential growth function.

[Color figure can be viewed at wileyonlinelibrary.com]

the first and second filtration the fouling layer diffuses back toward the center of the lumen leading to a larger initial algi nate concentration in the second filtration. Whereas the liquid in the lumen was exchanged via cross flow setup between the second and the third filtration. When modeling and fitting in analogy to the previously described experiments, $h$ was deter mined as a function of filtration time for all three filtration processes (Figure 5b). The overall behavior is the same, while the time scales slightly differ, but are in the same order of magnitude. The final value $h(t \rightarrow \infty)$ slightly increases with the number of filtration processes as expected. The small ratio $h_{1}(t \rightarrow \infty) / h_{3}(t \rightarrow \infty)$ of about $14 \%$ indicates, however, that the reversibility is good, but not perfect. To a large extent the fil tration of sodium alginate without $\mathrm{Ca}^{2+}$ can be regarded as a a)

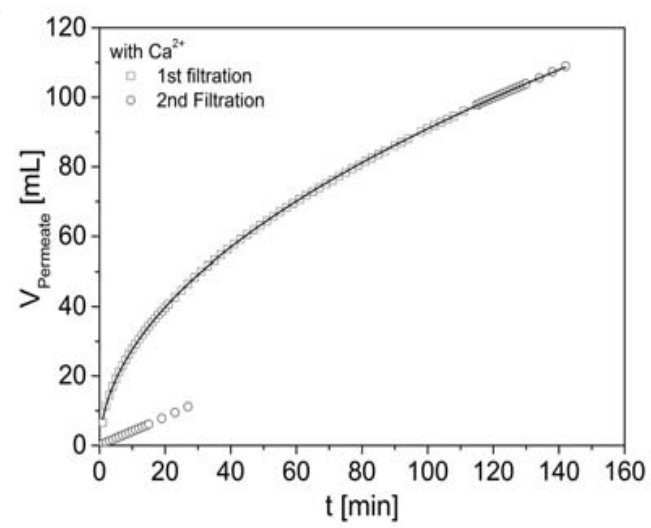

b)

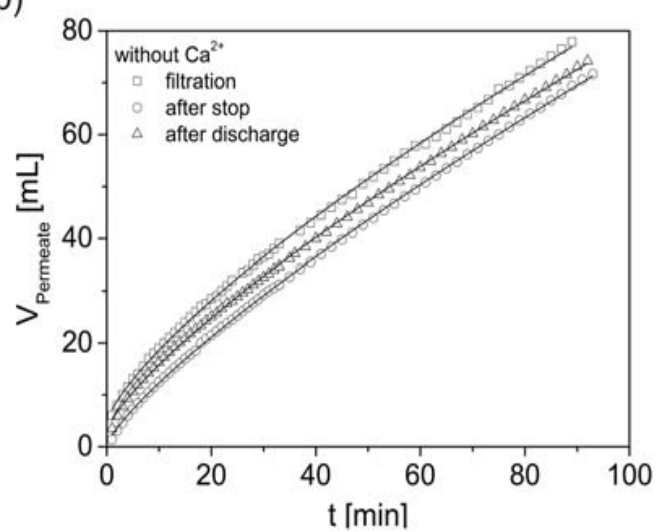

Figure 6. (a) Permeate volume $V_{\text {Permeate }}$ as a function of filtration time $t$ for sodium alginate filtration with $\mathrm{Ca}^{2+}$. The expected square root dependence of $V_{\text {Permeate }}(t)$ was observed. (b) In the filtration of sodium alginate filtration without $\mathrm{Ca}^{2+} V_{\text {Permeate }}(t)$ can be described empirically by $V_{\text {Permeate }}(t) \propto t^{0.7-0.9}$. 
a)

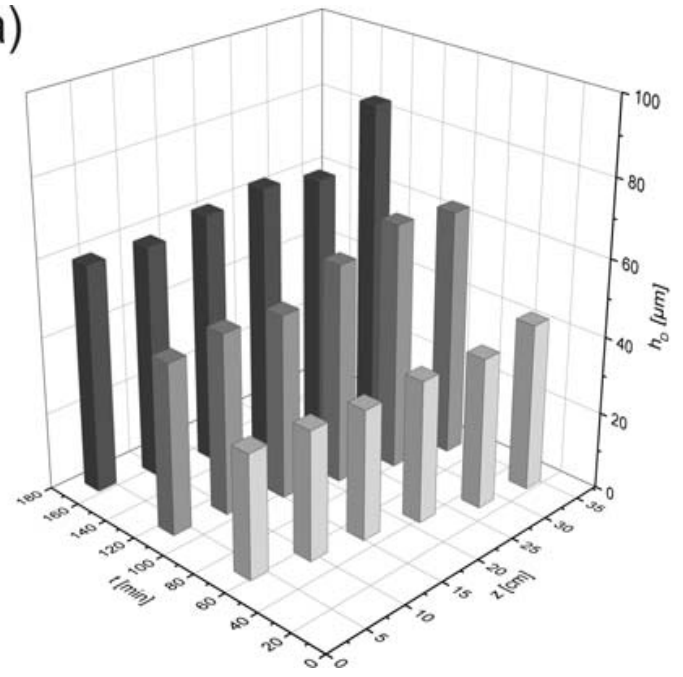

b)

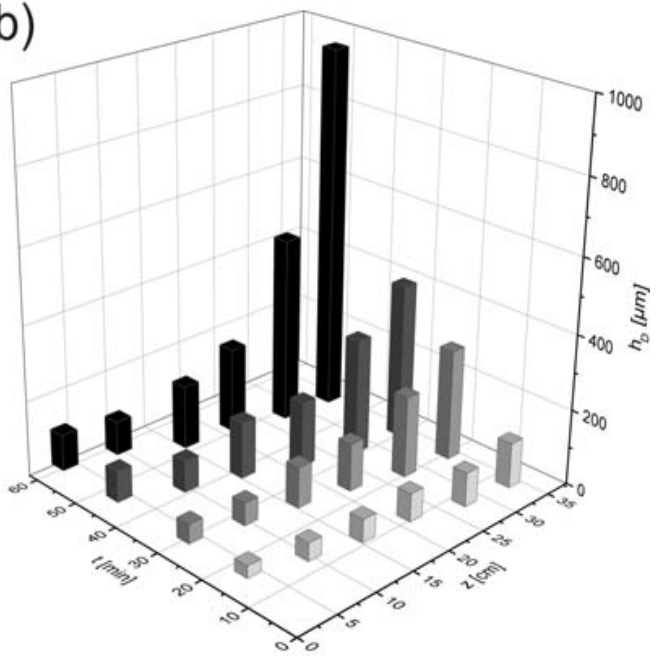

Figure 7. $h$ as a function of filtration time $t$ and $z$, that is, along the long axis of the cylindrical fiber.

(a) Ultrafiltration of aqueous sodium alginate solution with $\mathrm{Ca}^{2+}$ : the deposit layer is more or less equally distributed and grows with time. (b) The concentration polarization layer build-up is a strong function of $z$ and $t$ in the case of filtration without $\mathrm{Ca}^{2+}$.

reversible concentration polarization, whereas the filtration of sodium alginate with $\mathrm{Ca}^{2+}$ showed the characteristics of a dense gel layer formation. ${ }^{28}$ This detailed characterization was possible by the CS RARE MRI, revealing the characteristics of the filtration processes in great detail.

\section{Flux after interruption of ultrafiltration}

To underline the MRI data analysis, the permeate flux was integrally observed during the experiments. The ultrafiltration processes with and without $\mathrm{Ca}^{2+}$ were interrupted, and the pressure was reduced to 0 bar. After observation of the relaxa tion of the deposit, both filtrations were started again with the same filtration parameters. During these experiments, the per meate volume was volumetrically measured as a function of filtration times (Figure 6).

The flux is different in both experiments. For the filtration without $\mathrm{Ca}^{2+}$, an almost complete flux recovery is observed. The minimal deviations in the beginning of the three filtration processes show up in the measured volumes and might be attributed to pore blockage or small alginate agglomerates sticking to the membrane surface. The permeate volume for the filtration with $\mathrm{Ca}^{2+}$ behaves differently: In the second fil tration after the interruption, the flux increases monotonically and uniformly in continuation of the end of the first filtration.

These volumetric and integral results are in good agreement with the MRI measurements, where a compact and irreversible layer could be measured for the filtration with $\mathrm{Ca}^{2+}$ (Figure 4a) and a loose, fluffy layer with a larger extent could be observed for the filtration without $\mathrm{Ca}^{2+}$ (Figure 5a).

\section{Deposit formation along the fiber axis}

As the pressure varies along a ceramic hollow fiber mem brane, the deposit is expected to be a function of length, too. The coordinate is denoted as $z$ according to the NMR nomen clature and is 0 at the inlet of the feed into the fiber (Figure 1). The point at $z \quad 35 \mathrm{~cm}$ is located near the permeate outlet. In additional experiments, ultrafiltrations of sodium alginate with and without $\mathrm{Ca}^{2+}$ were investigated as a function of $z$ and of filtration time $t$. The MRI data were recorded and processed applying the abovementioned procedures. As the filtration mechanisms depend on the $\mathrm{Ca}^{2+}$ concentration, the $z$ dependence is expected to differ for these two experiments (Figures 7a, b).

In the case of gel layer formation (Figure 7a), the height of the deposit is again much smaller compared to concentration polarization (Figure 7b), but the increase as a function of $z$ is still pronounced and measurable. With increasing filtration time, the gel layer thickness increases slightly.

The $z$ dependence of the concentration gradient is much more pronounced in the filtration without $\mathrm{Ca}^{2+}$ : The deposit increases drastically with increasing $t$ and $z$, which was also reported by Panglisch in simulations of particle filtration in dead end mode. ${ }^{29}$ Please note the scale of $h$. The findings are in agreement with the expectation from classic filtration experiments and is now quantified noninvasively and time resolved during filtration experiments. Concluding, the experi ments show the capability of fast MRI to detect deposits in CHFMs and to provide the basis for quantification of typical time and length scales involved in filtration processes. Based on the spatially and time resolved MRI results the mechanisms can be described in detail which could lead to further develop ment of dedicated numerical and analytical models in filtration theory.

\section{Conclusions}

In situ filtration MRI experiments were quantitatively ana lyzed to describe the mechanisms of deposit formation during ultrafiltration of sodium alginate in CHFMs. The CS RARE pulse sequence allowed short measurement times while using relatively large repetition times to avoid inflow artefacts espe cially in the feed channel in the images. The membrane lumen was mapped in the images by angular averages of the intensity as a function of the radius. The radial intensity profiles were modeled to obtain the characteristic length scales of the deposit $h . h(t)$ could further be modeled with an exponential growth function with the characteristic time constant $t_{c}$. This parameter depends strongly on the $\mathrm{Ca}^{2+}$ concentration indicat ing the fouling mechanisms of the two ultrafiltration pro cesses. Low values for $t_{c}$ are attributed to concentration polarization and large values of $t_{c}$ indicate a dense and com pact, irreversible gel layer formation. This interpretation was underlined by flux interruption experiments, which gave 
insight into the reversibility of the deposit structures. As the deposit formation depends on pressure a height dependence is expected in both cases of filtration and is observed in MRI. Using the same modelling of the data, the deposit height was measured as a function of filtration time and fiber height $z$. The results are in agreement with the expectations from the postulated filtration mechanisms.

\section{Notation \\ Symbols}

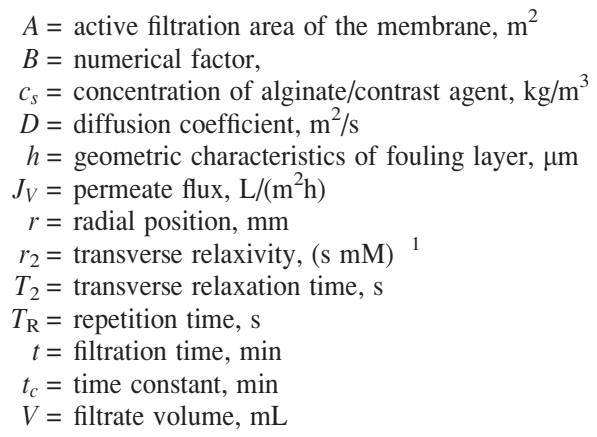

\section{Greek letters}

$$
\begin{aligned}
\sigma & =\text { standard deviation, } \\
\lambda_{1} & =\text { regularization parameter, } \\
\lambda_{2} & =\text { regularization parameter, } \\
\tau_{E, \text { effective }} & =\text { effective echo time, ms }
\end{aligned}
$$

\section{Acknowledgment}

The authors thank the German Research Foundation (Deut sche Forschungsgemeinschaft, DFG) for financial support of the instrumental facility Pro $^{2} \mathrm{NMR}$ and the MRI CFD project (GU1123/5 1). F. Ranzinger is thanked for assistance during the MRI measurements. A. Bakandritsos, A. Kolokithas Ntoukas and Y. Sarigiannis (Materials Science Department, University of Patras) are acknowledged for providing the MION based contrast agents (MagAlg and MagAlgPEG).

\section{Literature Cited}

1. Association AWW. Microfiltration and Ultrafiltration Membranes for Drinking Water. AWWA Manual M53. Denver, CO: Americal Water Works Association; 2005.

2. Li K. Ceramic Membranes for Separation and Reaction. Wiley, 2007.

3. Membrane Science and Technology Industrial, Biological, and Waste Treatment Processes. Springer, 1970.

4. Butehorn S. Experimental and Numerical Investigation of the Hydro dynamics of Microfiltration Processes Using a Multi Scale Approach. Aachen, Rheinisch Westfalischen Technischen Hoch schule Aachen; 2011.

5. Buetehorn S, Utiu L, Kuppers M, Blumich B, Wintgens T, Wessling M, Melin T. NMR imaging of local cumulative permeate flux and local cake growth in submerged microfiltration processes. J Memb Sci. 2011;371(1 2):52 64.

6. C, ulfaz PZ, Buetehorn S, Utiu L, Kueppers M, Bluemich B, Melin $\mathrm{T}$, Wessling M, Lammertink RGH. Fouling behavior of microstruc tured hollow fiber membranes in dead end filtrations: critical flux determination and nmr imaging of particle deposition. Langmuir. 2011;27(5):1643 1652.

7. Creber SA, Pintelon TRR, von der Schulenburg D, Vrouwenvelder JS, van Loosdrecht MCM, Johns ML. Magnetic resonance imaging and 3D simulation studies of biofilm accumulation and cleaning on reverse osmosis membranes. Food Bioprod Process. 2010;88(4):401 408.

8. Fridjonsson EO, Vogt SJ, Vrouwenvelder JS, Johns ML. Early non destructive biofouling detection in spiral wound RO membranes using a mobile earth's field NMR. J Memb Sci. 2015;489:227 236.
9. Valladares Linares R, Fortunato L, Farhat NM, Bucs SS, Staal M, Fridjonsson EO, Johns ML, Vrouwenvelder JS, Leiknes T. Mini review: novel non destructive in situ biofilm characterization techni ques in membrane systems. Desalination Water Treat. 2016;57(48 49):22894 22901

10. Pintelon TRR, Creber SA, von der Schulenburg DAG, Johns ML. Validation of 3D simulations of reverse osmosis membrane biofoul ing. Biotechnol Bioeng. 2010;106(4):677 689.

11. von der Schulenburg DAG, Vrouwenvelder JS, Creber SA, van Loosdrecht MCM, Johns ML. Nuclear magnetic resonance microscopy studies of membrane biofouling. J Memb Sci. 2008;323(1):37 44.

12. Vrouwenvelder JS, von der Schulenburg DAG, Kruithof JC, Johns ML, van Loosdrecht MCM. Biofouling of spiral wound nanofiltra tion and reverse osmosis membranes: a feed spacer problem. Water Res. 2009;43(3):583 594.

13. Airey D, Yao S, Wu J, Chen V, Fane AG, Pope JM. An investiga tion of concentration polarization phenomena in membrane filtration of colloidal silica suspensions by NMR micro imaging. J Memb Sci. 1998;145(2):145 158.

14. Pope JM, Yao S, Fane AG. Quantitative measurements of the con centration polarisation layer thickness in membrane filtration of oil water emulsions using NMR micro imaging. J Memb Sci. 1996; 118(2):247 257

15. Yao S, Costello M, Fane AG, Pope JM. Non invasive observation of flow profiles and polarisation layers in hollow fibre membrane filtra tion modules using NMR micro imaging. J Memb Sci. 1995;99(3): 207216.

16. Yao S, Fane AG, Pope JM. An investigation of the fluidity of con centration polarisation layers in crossflow membrane filtration of an oil water emulsion using chemical shift selective flow imaging. Magn Reson Imaging. 1997;15(2):235 242.

17. Seymour JD, Gage JP, Codd SL, Gerlach R. Magnetic resonance microscopy of biofouling induced scale dependent transport in porous media. Adv Water Resour. 2007;30(6 7):1408 1420.

18. Lustig M, Donoho D, Pauly JM. Sparse MRI: The application of compressed sensing for rapid MR imaging. Magn Reson Med. 2007; 58(6): 11821195 .

19. Arndt F, Schuhmann S, Guthausen G, Schutz S, Nirschl H. In situ MRI of alginate fouling and flow in ceramic hollow fiber mem branes. J Memb Sci. 2017;524:691 699.

20. Arndt F, Roth U, Nirschl H, Schutz S, Guthausen G. New insights into sodium alginate fouling of ceramic hollow fiber membranes by NMR imaging. AIChE J. 2016;62(7):2459 2467.

21. Arndt F, Heidebrecht H J, Schork N, Schuhmann S, Kulozik U, Schutz S, Nirschl H, Guthausen G, Deposit layer formation during skim milk dead end filtration with ceramic hollow fiber mem branes using magnetic resonance imaging. In: van Duynhoven J, Guthausen G, editors. Proceedings of the XIII International Con ference on the Applications of Magnetic Resonance in Food Sci ence. MR in Food 2016: impublications; 2016:55 59.

22. Zoppellaro G, Kolokithas Ntoukas A, Polakova K, Tucek J, Zboril R, Loudos G, Fragogeorgi E, Diwoky C, Tomankova K, Avgoustakis K, Kouzoudis D, Bakandritsos A. Theranostics of epi taxially condensed colloidal nanocrystal clusters, through a soft bio mineralization route. Chem Mater. 2014;26(6):2062 2074.

23. Ebrahimi M, Kerker S, Daume S, Geile M, Ehlen F, Unger I, Schutz $\mathrm{S}$, Czermak P, Innovative ceramic hollow fiber membranes for recy cling/reuse of oilfield produced water. Desalin Water Treat. 2015; 55(13):3554 3567.

24. Callaghan PT. Principles of Nuclear Magnetic Resonance Micros copy. New York: Oxford University Press, 1991.

25. Kanj R, Joshi R. Importance sampling based estimation: applications to memory design. In: Singhee A, Rutenbar RA, editors. Extreme Statistics in Nanoscale Memory Design. Springer US, 2010:51 96.

26. Lustig M. Compressed sensing MRI resources, software, 2016. Available at: https://people.eecs.berkeley.edu/ mlustig/Software.

27. Porter MC. Concentration polarization with membrane ultrafiltration. Product R\&D. 1972;11(3):234 248.

28. Reihanian H, Robertson CR, Michaels AS. Mechanisms of polariza tion and fouling of ultrafiltration membranes by proteins. J Memb Sci. 1983;16:237 258.

29. Panglisch S. Formation and prevention of hardly removable particle layers in inside out capillary membranes operating in dead end mode. Water Sci Technol Water Supply. 2003;3(5 6):117 124. 


\section{Repository KITopen}

Dies ist ein Postprint/begutachtetes Manuskript.

Empfohlene Zitierung:

Schuhmann, S.; Schork, N.; Beller, K.; Nirschl, N.; Oerther, T.; Guthausen, G. In-situ characterization of deposits in ceramic hollow fiber membranes by compressed sensing RARE-MRI.

2018. AIChE journal, 64.

doi: $10.5445 / / R / 1000088458$

Zitierung der Originalveröffentlichung:

Schuhmann, S.; Schork, N.; Beller, K.; Nirschl, N.; Oerther, T.; Guthausen, G. In-situ characterization of deposits in ceramic hollow fiber membranes by compressed sensing RARE-MRI.

2018. AIChE journal, 64 (11), 4039-4046.

doi:10.1002/aic.16201 